\title{
Synergy of Intellectual Property and Traditional Knowledge: Holy Grail for Protection and Sustainable Future
}

\author{
Dipak B. Shukla*
}

\author{
Business Development \& Information Management Division, Central Salt \& Marine Chemicals Research Institute \\ (CSIR), G.B.Marg, Bhavnagar-364 002, Gujarat, India
}

\begin{abstract}
The symbiosis of Intellectual Property Rights (IPR) and Traditional Knowledge (TK) has become indispensable for its creators and for the world's intellectual community at large. Evidently, the need for preservation, protection and promotion of TK has become inevitable for self-sustenance, economic prosperity of knowledge holders and competitive business advantage. Obviously, the promotion of TK is now widely recognized and it plays an eminent role in supporting TK-based community's livelihood and cultures. The exponential growth of TK has galvanized new forms of IP protection, especially for traditional medicine (TM). The traditional healthcare problems, complexities linked to IP in TK, and community knowledge are posing a gargantuan challenge to sustainable development, intellectual and cultural vitality.

This paper stresses on the scope of TK, the avenues to protect it under the existing IPR regime. It incisively highlights the challenges faced by TK holders and the subsequent need for its protective regulation. The paper enunciates the Traditional Knowledge Digital Library (TKDL) as a versatile global regulatory device to obviate the usurpation of documented knowledge. It imparts the vital prerequisites for TKDL and delineates the crucial role it plays, in preserving prized heritage and acceleration of modern research on Ayurveda. The synergy of IPR and TK continues to be nebulous but empirical evidence suggests the rationale for protecting TK within the IPR ambit. The sine qua non of TK protection is to tackle the major concerns like equity, conservation, preservation of traditional culture, bio-piracy, promotion and evolution of TK. The cardinal focus here is the urgent need for international coordination and cooperation to efficaciously safeguard and leverage TK. Any protective strategy should take into account the ethnic community and the attendant global dimensions. The bottom-line is that such a protection should be effectual, comprehensible and accessible to TK holders. Interfacing IPRs with TK rights will only pave the way for TK protection, knowledge enrichments on TM, wellarticulated human resources creation and nourishment of TK culture.
\end{abstract}

Keywords: Intellectual Property Rights (IPR), Traditional Knowledge (TK), traditional medicine (TM), Traditional Knowledge Digital Library (TKDL).

\section{INTRODUCTION}

The twenty-first century will be the century of knowledge, indeed a century of the mind. A nation's ability to convert its traditional knowledge (TK) into wealth and social good through the process of protection and promotion will determine its future and concomitantly its sustainability. In this context, issues of preservation, recognition for knowledge holders and documentation of traditional medicine (TM) will become critically important all around the world. The effective protection and promotion of TM and its use, increasing demands for new forms of IP protection for $\mathrm{TM}$, as well as access to TK related information, increasing dominance of TM over allopathic medicine are posing challenge in setting a new $21^{\text {st }}$ century IP agenda. Nurturing a strong TK base, particularly the great strength in $\mathrm{TM}$, through a balanced system of statutory recognition and rewards is the need of the hour. In the present global scenario, the need for preservation, protection and promotion

*Address correspondence to this author at the Business Development \& Information Management Division, Central Salt \& Marine Chemicals Research Institute (CSIR), G.B.Marg, Bhavnagar-364 002, Gujarat, India; Tel: 2567466 ext. 788; E-mail: dbhsukla@csmcri.org of TK including TM have become imperative for selfsustenance, economic growth of knowledge holders and competitive business advantages.

In this new millennium, the international fora on intellectual property rights (IPRs) strongly recommended that TK must be an intrinsic ingredient of IPR system. TK is an enormous oasis and its easy accessibility made it susceptible to misappropriation. The reliability of TM systems coupled with the absence of such information with international searching authorities (Patent Offices) provided an easy opportunity for interlopers to get patents on yoga accessories, yoga-related copyrights, yoga trademarks, and on therapeutic formula derived from TM systems. The grant of patents on non-original inventions particularly TM, have been a cause of great concern to the developing countries which holds the knowledge. Numerous cases of bio-piracy have highlighted this issue and have increased demand for protection of TK from such misappropriation causing many biodiversity rich countries to design and adopt different protective regimes. At the international level there is a significant support for opposing the grants of patents on nonoriginal inventions. For example in 1997, CSIR (India) challenged the US patent No. 5,401,504 on wound healing 
properties of turmeric. The Indian Officials produced ancient Sanskrit texts and a paper [1] published in 1953 to support their claim that the use of turmeric for medical purposes was known in India for many years and hence its use as a medicine was not new invention. The objection was upheld and the patent was cancelled on the grounds that it failed to meet the novelty criteria. In another landmark event in May 2000, the EP patent No. 436,257 on method for controlling fungi on plants by hydrophobic extract of neem seeds, issued to W.R. Grace Company and US Department of Agriculture, was revoked [2] and quashed on grounds that the innovation did not fulfill the criteria of novelty and inventive step. The extract having fungicidal effect was part of Indian indigenous knowledge and was used for Ayurveda cure for dermatological ailments and protecting crops from fungal infection. In yet another case of post grant opposition on non-original invention in TK systems was the filing for reexamination of US patent No. 5,663,484 on basmati rice lines and grains by Govt. of India. The latter provided written proof from US National Agricultural Statistical Service 1998 which stated that $25 \%$ of the total rice import was from India and these varieties cannot be grown in the US. This piece of information was submitted to the USPTO which became a vital weapon for India to challenge the patent that this plant varieties and grains already existed in India. The Ricetec company had to withdraw some claims and also some dependants claim.

To mitigate these types of problems, the Govt. of India has created a traditional knowledge digital library (TKDL) on traditional medicinal plants and systems, [3] which will also lead to a traditional knowledge resource classification (TKRC). The latter has been accepted and linked to International Patent Classification (IPC) system which will pave the way of building a bridge between the knowledge contained in thousands of old Sanskrit, Arabic, Persian, Urdu and Tamil books and manuscripts and the computer screen of the international searching authority. This will eliminate the problem of grant of wrong patents since the examiners will be aware of rights of that knowledge which existed for age old centuries.

In recent year, science in Western countries has been giving more impetus to TM. They have realized that TM has the real panacea to the current healthcare problems, sometimes in sync with modern S\&T knowledge. Notwithstanding the growing recognition of TM as a valuable source of knowledge, it has been regarded under western IP laws as "Information" in public domain which can be freely used by anybody. Moreover, in some cases, diverse forms of TM have been appropriated under IPRs by researchers and commercial enterprises without any compensation to the knowledge creators or possessors. Any attempt to exploit TK for industrial or commercial benefit may lead to prejudicial misappropriation of the same from its rightful holders. Hence, it becomes pertinent to develop ways and means of protecting, strengthening and nurturing TK thereby ensuring sustainable development compatible to the interest of the TK holders. TK coupled with IPR will provide pathways to social and economic developments and the fruits can be enjoyed by the posterity. Thus, the traditional communities will continue to thrive and develop in different ways consistent with their values and interest.

\section{THE SCOPE OF TK}

World Intellectual Property Organization (WIPO) a specialized agency of UN, has defined TK [4] as "traditionbased literary, artistic or scientific works, performances, inventions, scientific discoveries, designs, marks, names and symbols, undisclosed information and all other traditionalbased innovations and creations resulting from intellectual activity in the industrial, scientific, literary or artistic fields." In light of this definition it can be inferred that TK is vast enough to encompass knowledge related to various categories like agricultural-knowledge, medicinal knowledge, biodiversity-related knowledge and expressions of folk fore in the form of music, dance, song, handicraft, stories and art work.

TK encompasses different types of knowledge. These may be distinguished by the elements involved like the knowledge's potential or actual applications; the level of codification; the individual or collective form of possession and the legal status (can be intellectual property). The desire to protect TK has generated a significant body of literature and many proposals of regulation and for action in different international fora [5]. TK includes information on the use of biological and other materials for medical treatment and agriculture, production processes, designs, literature, music, rituals and other techniques and arts. This broad set includes information of a functional and of an aesthetic character i.e. processes and products than can be used in agriculture or industry, as well as intangibles of cultural value.

TK comprises of knowledge which has been evolved in the past, and will still continue to develop in the new millennium. Most TK including TM is non-contemporary in nature; it has been used for generation together and in many cases collected and published by anthropologists, historian, botanists or other researchers and observers [6]. However, TK is not static; it evolves and generates new information as a result of improvement or adaptation to changing circumstances. The context of TK varies significantly and its forms of expression. Some TK is codified i.e. formalized in some way (e.g. textile designs, Ayurveda, traditional medicine). A great part of TK is non-codified or tacit such as "Folk", "Tribal" or "Indigenous" medicine, which is generally based on traditional creed, norms and practices accumulated during centuries old experiences of trial \& error, success \& failures at household level and passed on to successive generation through oral tradition.

TK is generally possessed by individuals (e.g. healing practices and rituals), by some members of a group or be available to all the members of a group (common knowledge). Take for example, knowledge on herbal home remedies is held by millions of women and elders. Days are not far away when TK-based products will be available in open market through commercial outlets and the millions of TK holders stand to gain from the economic recompense in exchange of their knowledge utilized. Some TK is used and understood outside its local/traditional communal context, but this is not always the case. There are several spiritual components in TK characteristic to each community. Knowledge that cannot be leveraged or capitalized beyond its communal context has little or no commercial relevance. The bottom line is, TK includes information of different kinds and functions, developed in ancestral times but is 
subject to contemporary improvement and adaptation. It is expressed in several documented and non-documented forms and may have commercial value depending on its potential or actual use.

\section{India's Initiatives for Documentation of TK}

The paramount importance and value of protecting India's TK was envisaged way back in 1996 in the aftermath of the landmark victory in the Battle of Haldighati. For the first time, US patent No: 5,401,504 on TK (turmeric patent) granted by USPTO was challenged by India and the patent was revoked. This was followed by neem, basmati, bittergourd, jamun, karela and other traditional remedies. The enormous efforts put in by the Indians (IP officers, IT professionals, Ayurveda experts, scientists) in terms of man hours, money and material and the success in turmeric case have led to the acceptance of TK as a vital component of the Industrial Property System. The salient feature in the area of TK, especially protecting the traditional medicine (TM) and other genetic resources emphasized the crying necessity of creating a TKDL on Ayurveda i.e. "Science of Life" which is based on traditional knowledge resource classification (TKRC). In 2001, the International Patent Classification (IPC) Union of WIPO endorsed the concept of traditional knowledge digital library (TKDL) and in February 2003, the IPC union decided to create a new sub-class for Traditional Knowledge Resource Classification (TKRC) and fuse TKRC with Ayurveda [7]. TKRC is an innovative structured classification system that enables the retrieval of information on TK in a scientific and rational manner for patent examiners. The TKDL initiative was spearheaded by the Department of Indian Systems of Medicine and Homeopathy (ISMH) which had set up an inter-disciplinary task force to develop the digital database. The TKDL has documented the extant traditional medicinal knowledge available in public domain by shifting and collating information on TK from the existing disclosed literature covering Ayurveda. The TKDL will have a crucial role not only in documenting our precious heritage in the area of traditional health-care systems but also enable patent offices all over the world to search and examine any prevalent use/ prior art, thereby preventing biopiracy and unscrupulous patenting of herbal medicine formulation. In a recent development an international agreement [8] with European Patent Office (EPO) has been established and the maiden Indian effort in creating the TKDL would now be available to the patent examiners at EPO. This agreement is unique and would have long-term implication on the protection of TK including TM and global IP systems. The TKDL Access Agreement with EPO would pave the way for similar agreement with other international patent offices to prevent the misuse of vast information of huge economic potential in easy to access form.

In TKDL, the words "Traditional" and "Digital" are coming closer. Otherwise, they are like North Pole and South Pole i.e. Traditional meaning goes back to old centuries and Digital words is of recent origin meaning belonging to twenty-first century. The recent international scenario on TK, make it quintessential for these words to coalesce. The availability of TK in retrievable form and in many languages has given immense impetus to modern research in developing countries as it per se may get involved in pioneering research that would add value to TK.
It is élan vital because we should not just protect or prevent others from misusing the knowledge, but we must ourselves, being the privy to this knowledge, add value to it by doing further research, and hence the advent of modern knowledge becomes extremely important.

\section{TKDL - A Vital Prerequisite}

It is immensely important and indispensable for IP officers, IT professionals, Ayurveda experts, international patent examiners, research scholars on traditional medicine, R\&D personnel's to have explicit knowledge on enormous literature on Ayurveda, yoga, traditional therapeutic formula that has been included in the digital library. The digital and computerized database will play a crucial role in

* documenting our prized heritage in the area of traditional health care systems

* establishing our rights and claims on medicinal knowledge of plants and validate it.

* preventing grant of patents on Ayurvedic drugs and save huge amount of money and time needed for contesting the patents filed on TK claiming for nonoriginal invention.

* accelerating modern research on Ayurveda leading to higher acceptability at international level and will give significant impact to export of Indian Herbal Products.

* documenting the scattered information on Indian systems of medicine, particularly in a lingua franca which is easily understood by patent examiners.

* integrating widely dispersed and distributed references on TK system in a retrievable and accessible form and act as a bond between the traditional and modern knowledge systems.

* providing feedback mechanism on the coverage of divergent viewpoints and minimize controversies on herbal drugs in future.

\section{TKDL - A Tool to Prevent Misappropriation of TK}

The humble purpose of establishing TKDL [3] is to assimilate the scattered and non-documented literature on TK and bring it into a format which can be easily accessed, understood and retrieved by International Patent Examiners.

In the TRIPS agreement of WIPO, the classification system in IPC for the documentation of TK, had only one single subgroup related to medicinal plants. Therefore, a modern classification system was evolved which was in line with IPC. Agencies like Indian National Institute of Science Communication and Information (NISCAIR-CSIR) and Dept. of Indian Systems of Medicine and Homeopathy, Govt. of India left no stone unturned and prepared a classification system especially for Ayurveda, in the first instance, and named it as Traditional Knowledge Resource Classification (TKRC). The basic concept of TKRC and the novel aspects of the classification scheme was presented to the experts of the task force committee having officials from USPTO, EPO, China, Japan and India. After meticulous deliberations, the committee recognized the need of having a more detailed classification relating to medicinal plants. A draft recommendation was submitted and unanimous 
consensus was given to include ca. 200 subgroups against earlier few subgroups on medicinal plants [9] and linked TKRC to IPC. A new main group was included in IPC i.e. A61K 36/00 with 207 subgroups covering different categories of plants. In February 2003, IPC Union took a decision (i) to create a new sub-class for TKRC, (ii) to link TKRC with IPC and (iii) to continue to work on biodiversity, TK and traditional cultural expressions (TCEs). This will have significant impact on the system of search and examination while granting patents in the area of TK whereby the possibilities of granting of wrong TK patents will be significantly reduced.

TKDL targets Indian Systems of Medicine i.e. Ayurveda, Unani, Siddha, Yoga and Naturopathy available in public domain. The related information was documented from the existing literature available in Sanskrit, Urdu, Arabic, Persian and Tamil languages. This was translated into five international languages like English, German, French, Spanish and Japanese which could be understood by international patent examiners. TKRC is an innovative structured classification system for the purpose of arrangement, dissemination and retrieval of TK. As per the IPC rules, [10] the information is classified under sections, subclass, subgroups which makes it easy for patent examiners to access. At present ca 0.2 million formulations has been transcribed for realizing the objective of TKDL project.

TKDL - is basically a software and with its classification system converts text in local languages into multiple international languages. This software does not transliterate, but it does a knowledge based conversion where the abstract is converted into several languages using Unicode, metadata methodology. This software also converts traditional terminology into modern terminology e.g. Turmeric to Curcuma longa Linn; Neem to Azadirachta indica A. Juss; Basmati rich to Oryza sativa Linn; Jwar to fever; Kumari to Aloe vera and Mussorika to small pox. TKDL includes search interface which provide full text search and retrieval of TK information on IPC and keywords in multiple languages. The search features includes single or multiple word search, Boolen expression search, field search, phrase search etc. Searches are also available on IPC and TKRC codes.

TKDL will act as a bridge between local languages and patent examiners at a global level. The database will provide information on modern as well as local names in a language and format which will be understood by patent examiners. It is expected that TKDL will address and narrow down the gap on lack of access to prior art on traditional knowledge. It also seeks to give recognition and legitimacy to the present TK and enable protection of knowledge from getting patented.

Today, India through TKDL is capable of protecting about 0.2 million medical formulations similar to turmeric, neem, karela, jamun etc. On an average it takes 5 to 7 years to oppose a granted patent at international level which may cost ca 0.4 million US\$. This works out that the cost of protecting 0.2 million medicinal formulations, in absence of TKDL, would be staggering and unaffordable. The TKDL
Access Agreement [8] with EPO on 2 February 2009 would enhance the negotiating strengths of India and developing countries at international fora. In fact, the international IP community has recognized TKDL as an effective tool for defensive protection of TK.

\section{Problems Confronting TK Holders}

The TK and indigenous traditional knowledge holders in developing countries are always looked upon as frolicsome. In most of the communities, TK is inseparable from their very ways of life and their cultural values, spiritual beliefs and customary legal systems. These communities lack in academic knowledge and are remote to the recent developments taking place globally. This and many other facts deter them from knowing their rights or the economic value of the traditional knowledge possessed by them. Some of the main problems faced by them are

\section{i. Means to Revitalize and Nurture TK}

One of the vital problems faced by TK holders is to find ways to strengthen and nurture the roots of TK globally. With adequate legal system of strengthening, the fruits of TK can be actualized by future generation akin to IPRs where the legal heirs have equal rights. With this type of protection, the traditional communities will pursue, thrive and develop knowledge which will be consistent with their own values and domain. TK and TM holders are zealous to ensure that their knowledge should not be misused by others without their consent. There should be provision for fair sharing of the benefits. This will ultimately lead to greater respect and recognition for the values, contributions and concerns of TK holders.

\section{ii. Apathetic Attitude and Lack of Respect}

Another difficulty faced by TK holders is the lack of respect and appreciation for the knowledge possessed. For example, a traditional medical healer gives a concoction of herbs to cure an illness. His medical treatment is empirically inherited from the clinical trials undertaken in past and on solid understanding of the interplay between the mixture and human physiology. In absence of academic knowledge, the healer may not be able to isolate the chemical compounds or describe its effect on the body in terms of modern biochemistry. Sometimes, the true understanding of the value of TK remains elusive when the scientific and technical qualities are viewed from a parochial and skeptical perspective. Recent reports reveal that many consumers in developed countries use TM for treating illness with an understanding that such alternative or complementary systems are firmly routed on empirical assumptions over many generations.

\section{iii. Commercial Exploitation and Benefit Sharing}

Yet another problem encountered by TK holder is the exploitation of their knowledge by other unscrupulous elements of the society. Also the benefits accrued, in case of commercial exploitation are not equally shared. This gives rise to probable questions like: (i) what is the legal protection of TK against its misuse? (ii) what is the role of prior informed consent? and (iii) why there is a need of equitable benefit-sharing? All this needs a quick perusal. A lack of experience with present formal systems, limited economic 
resources, lack of unified voices and clear national policy related to utilization and protection of TK would result in obvious disadvantage in using the existing IP mechanism of TRIPS agreement. Furthermore, the lack of clear rules concerning the appropriate use of TK creates an atmosphere of uncertainty for R\&D scientists to use TK in development of new molecule effective on particular disease. This reflects that there is a common need for well articulated and predictable rules both for the holders and legitimate users of TK.

\section{Protection of TK Under Existing Modes of IPRs}

IPR can be looked upon as the best possible mode to protect TK. There are ardent proponents and opponents for extending IPRs to the knowledge of indigenous and traditional communities. One section which advocates the application of IPRs to TK, finds that TK can be protected by existing IP or by modifying certain aspects of the current forms of IPR protection. While the other group opposes the application of IPRs and is against modifying the present form of IPRs to protect TK with the excuse of incompatibility between the concepts of western IPR \& the practices and culture of local and indigenous communities in developing countries. But the bottom line should be that with all the inherent snags in establishing IPR protection for TM, the national IPR legislation and international conventions [11] should come forward to ensure that such knowledge is not held hostage to the parochial ideologies. Incontestably, we should endeavor to prevent its appropriation and straying away from the IPR system.

The possibility of applying different modes of IPRs protection to different components of TK is delineated below. It is observed that patents are granted on traditional medicine, natural components as well as on combinations of plants for therapeutic use [12]. However, since TK is not contemporary and is being used for long periods, the novelty and inventive steps requirements for patent protection are difficult to meet. Some valuable TK are kept secret e.g. applications of plants for therapeutic purpose. The traditional knowledge holders are protected against disclosure under unfair competition rules which do not require registration or other formalities. Most law require, as a condition for protection, [13] that information holder adopt steps to keep the information confidential. Conversely, there must be deliberate acts aimed to protect the relevant information, as secret. Geographical Indication (GI) is another suitable mechanism to enhance the value of agricultural products, handicrafts and other TK - derived products. Trademarks protect signs or symbols of commercial interest to local and indigenous communities. Copyrights and industrial designs provide a possible framework for the protection of various components of TK. Hence it can be said that IP and TK are the forms of creative endeavor and ancestral knowledge and tradition respectively that can be protected through existing modes of IPRs

\section{Copyright}

It is a powerful weapon to protect the artistic manifestation of TK holders, especially artists who belong to native communities against unauthorized reproduction and exploitation. It includes literary works such as tales, legends and myths, poems, traditions; textile works such as fabrics, garments, textile compositions, tapestries, carpets; musical and pictorial works and three dimensional works such as pottery \& ceramics, sculptures, wood and stone carvings and artifacts of different kinds. Besides this, rights to copy such as performance of singers and dancers, presentations of stage plays, puppet shows etc can be protected under this IPR system. CR protects only the way information is expressed and not the information itself.

\section{Inventions}

The different facets of TK including TM can be protected under the patent system. It can be used for protection of technical problems identified in prior art which are novel and have inventive steps on worldwide basis and has industrial application. For TK and genetic resources, patents can be filed for products isolated, synthesized from genetic structures, micro-organisms and plants or animals or organisms existing in nature. Under IPR systems, protection can be obtained for processes known to ethnic communities and tribes, which satisfy the criteria of novelty, inventive steps and utility [14]. All the results of biotechnology applied to genetic and biological resources and also some undisclosed techniques to obtain practical results can be hermetically protected with patents.

\section{Plant Varieties}

Under the plant breeders' right of the Convention of Biological Diversity (CBD), it is possible to protect new plant products, cultivars and varieties of all species of plant. In India, the Protection of Plant Varieties and Farmers' Right (PPVFR) [15] came into force in September 2001 which is consistent with the TRIPS of WTO. The new plant varieties in order to be protected must satisfy the criteria [16] of protection popularly abbreviated as DUS i.e. (i) the variety should be distinguishable (D) by one or more characteristic features from other varieties; (ii) it must be homogeneous or uniform (U) with regard to its vegetative propagation or sexual reproduction and (iii) the variety must be stable (S) after repeated propagation. The plant varieties leading to medicinal use can be legally protected under this IPRs system. Further, any improvement in this variety which represents the natural state of plant diversity also constitutes the new variety to be eligible for protection.

\section{Geographical Indication and Appellations of Origin}

It is a name or sign used on specific products which corresponds to a specific geographical location or origin. The use of Geographical indication (GI) will act as a certification that the product possess certain qualities or enjoy a reputation due to its geographical origin. India has enacted the GI of Goods Act 1999 [17] which states "An indication which identifies goods such as agri-goods, natural goods or manufactured goods as originating or manufactured in the territory of a country where a given quality or characteristics of such goods is essentially attributable to its geographical origin". There are situations where biological inventions would emanate from traditional practices or from established traditional products used locally. For example, biotech goods in relation to GI in the Indian context are Darjeeling tea, Basmati rice, Hyderabadi biryani, Bengali rasogulla, Gujarati dhokla, Bikaneri bhujia etc. The article 
22 of TRIPS of WTO clearly states that the rights under GI are community rights and not rights of individuals. The article also conveys that the benefits accrued from the protection of rights emanating from GI are to be passed on to the communities that are the rightful owners.

\section{Industrial Designs}

They are IP rights that make exclusive the visual designs of objects that are not pure utilitarian. It consists of creating a shape, configuration or composition of pattern or color in three-dimensional form containing aesthetic value. The design and shape of furniture, receptacles, garments, articles of ceramics, wood, leather etc prepared using indigenous knowledge of individual or the group of people in a traditional fashion qualifies for protection as industrial designs.

\section{Trademarks}

It is also one type of IP and is typically a name, word, logo, phrase, symbol, design, image or a combination of these elements. It is a distinctive sign or indicator used by an individual, business organization, craftsmen, indigenous communities (cooperatives, guilds, etc) or other legal entity to identify their products or services to consumers and to distinguish its products or services from those of other entities. It is an essential element of IP in the commercial promotion of goods and services globally.

\section{Repression of Unfair Competition}

Unfair competition conveys an act of competition which is contrary to honest practices in industrial or commercial matters and includes such acts that mislead public or cause confusion. This notion (indirect law) allows action to be taken for false or misleading claims that a product is authentically indigenous and it concerns a particular traditional community. Traditional secrets relate to ancestral knowledge possessed by native and indigenous communities and which has technological and economical value can be protected by provisions against unfair competition [18]. By this, undisclosed information can also be protected by repression of unfair competition law and thereby it will be possible to access and monitor that secret knowledge, its exploitation and communication by interested third parties. Protecting the secret TK through IPR system will enhance the scope of licensing of that secret knowledge which in turn will derive profit from its commercial use. After adequate repression of unfair use of TK it is essential to give wide publicity and keep abreast the indigenous communities, guild etc about the opportunities the secrecy regime offers for controlling the dissemination and exploitation of TK.

\section{Legislation to Protect TK}

In yesteryears, the issues of legislation on TK protection were debated in several international platforms under the auspices of Convention of Biological Diversity, the WTO, the WIPO which led to establishment of an Intergovernmental Committee on IP and Genetic Resources, TK and Folk fore and the UN Food and Agriculture Organization. Several policies were discussed to ensure that bio-prospecting must be regulated so that the stakeholders are benefited. The measures include access and benefitsharing legislation and egalitarian distribution of financial benefits with communities. Several countries including India, Costa Rica and member states of the Andean Community (Bolivia, Colombia, Ecuador, Peru and Venezuela) have included in their legislation that the patent application for inventions resulting from bio-prospecting expedition should invariably (i) indicate the source of biological material and (ii) include documentary evidence that the biological material was collected after the official consent of the providing country [19].

In India, patenting TK is not allowed under the Patent Law. According to section 3(P) of the Indian Patent Act, an invention which is a traditional knowledge or which is an aggregation or duplication of known properties of traditionally known component is not considered as an invention under the Act [20]. India is a party to CBD and has taken several steps to protect TK. The Act provides protection to biological diversity, sustainable use of its components and equitable benefit sharing arising from the use of biological resources. It also addresses the concerns like access to and collection and utilization of biological resources. The main intent of this legislation is to protect India's biodiversity and traditional knowledge [21] against its use by individuals/companies, corporations without sharing the benefits with possessors and also to check biopiracy.

Inter alia, India has made a maiden effort to document TK in a written as well as electronic form by means of People's Diversity Registers (PDR) [22] and the TKDL. TKDL was launched by Government of India on 26 March 2002 and very recently an agreement was made with EPO for facile access of documented TK. The main underpinning of the PBR was to build an open and transparent information system on biodiversity resources from the rural to urban remote area. This register can be used to promote sustainable management of biodiversity resources and also serve as a versatile tool to (i) document, monitor and provide information for sustainable management of local biodiversity resources; (ii) establish claims of individual and local communities over knowledge of uses of biodiversity resources; (iii) provide equitable share of benefits from the use of such TK and resources; and (iv) perpetuate and promote the development of practical ecological knowledge of ethnic communities and of traditional slow sciences such as Ayurveda, Unani and Siddha medicines.

\section{CONCLUSION}

Unlike the 'art for art's sake', protection for protection sake is not a self-sustaining proposition in the present knowledge era, which is characterized by fundamental human rights - proprietary and egalitarian. The importance of protecting the knowledge, innovations and practices of indigenous and local communities is increasingly recognized in several international forums. Developing countries in possession of traditional knowledge must ensure that the benefits of age-old knowledge, cumulative innovations associated with TK accrue to its holders while enhancing their socio-economic development for sustainable future. These countries also aim at preventing the improper appropriations of TK, with little or no compensations for the custodians of TK and without their prior informed consent. It is only in the new millennium that the TM protection and its 
documentation has been aggressively pursued by India and has created TKDL as a regulatory device to forestall biopiracy and unscrupulous patenting of herbal medicine formulations. Large number of patents filed globally on TM and traditional medicinal formulations/compositions caution the policy makers, knowledge holders, research scholars on Ayurveda, Siddha, Unani and ethnic communities that they need to be circumspect about leveraging all the existing forms of IPR for the protection of extant TK either as implicit knowledge or explicit knowledge. The tacit nature of a great deal of TK also makes the need for documentation of TM an urgent and pertinent issue.

Apathy or ignorance among knowledge holders and ethnic communities about TM protection impedes the very raison d'etre of drafting prior art documents supported by sound evidence and legalities. However, it is the moral responsibility of the policy makers to protect the empirical TK failing which an unscrupulous person could infringe and pirate the opportunities built through traditional knowledge. Quintessentially, both the knowledge holders and policy makers must ingest the vital components of preparation of TK documentation and synchronize this learning with the TM activities and practices. The bottom-line is sound interaction among developing countries on national strategies for strengthening TK, developing a sui generic system for the protection of TK and extensive commercialization of TM-based products and services.

\section{ACKNOWLEDGEMENTS}

The author gratefully acknowledges the grant from CSIR, New Delhi and Dr P.K. Ghosh, Director, CSMCRI Bhavnagar for his kind permission.

\section{ABBREVIATIONS}

IPRs $=$ Intellectual Property Rights

TK = Traditional Knowledge

$\mathrm{TM}=$ Traditional Medicine

TKDL $=$ Traditional Knowledge Digital Library

TKRC = Traditional Knowledge Resource Classification

IPC = International Patent Classification

WIPO = World Intellectual Property Organization

$\mathrm{EPO}=$ European Patent Office

IT = Information Technology

IP $\quad=$ Intellectual Property

TCE $=$ Traditional Cultural Expressions

USPTO $=$ United States Patent $\&$ Trademark Office

CSIR = Council of Scientific \& Industrial Research

TRIPS = Trade Related Intellectual Property System
GI $=$ Geographical Indication

CBD Convention of Biological Diversity

WTO World Trade Organization

\section{REFERENCES}

[1] Ismail, Z; Fakir, T. Trademarks or trade barriers?: indigenous knowledge and the flaws in the global IPR system. Int. J. Soc. Econ., 2004, 31(1/2), 173-194.

[2] Shiva, V. North-south conflicts in intellectual property rights. Peace Rev., 2000, 12(4), 501-508.

[3] Sen, N. TKDL- A safeguard for Indian traditional knowledge. Curr. Sci., 2002, 82(9), 1070-1071

[4] WIPO, The Protection of Traditional Knowledge. Overview of Policy Objectives and Core Principals. Document No WIPO/GRTKF/IC/7/5. 20 August 2004.

[5] Dutfield, G. Intellectual property rights, Trade and Biodiversity. London: IUCN and Earth scan, 2009, p. 239.

[6] Rao, V. Traditional knowledge: Commercialization-benefit sharing. Presentation at the International Seminar on Systems for the Protection of Traditional Knowledge, UNCTAD, New Delhi. 3-5 April 2002.

[7] www.tkdl.res.in/tkdl/langdefault.common/abouttkdl.asp

[8] CSIR News. 28 February 2009, 59(4).

[9] Convention on Biological Diversity. UNEP/CBD/WGABS/5/INF/6 26 Sept. 2007. Biodiversity and the Patent System: Towards International Indicators, Understanding the IPC p.11

[10] www.wipo.int/classifications/ipc/en/general

[11] Gutter man, A. Innovation and Competition Policy: A comparative study of regulation of patent licensing and collaborative research \& development in the US and the European community, Kluwer Law International, London 1997.

[12] Locke, J.C.; Larew, H.G.; Walter, J.F. Method for controlling fungi on plants by the aid of hydrophobic extracted neem oil. EP publication No: 04, 36,257 and EP application No: 99, 02,50,319.2 20 December 1990.

[13] Section 7 of TRIPS Agreement. Protection of undisclosed information.

[14] Criteria for patentability. Novelty, Inventive steps and Utility. Article 27 in Section 5 of the TRIPS Agreement. "Patentable Subject Matter"

[15] Bala Ravi, S. MSSRF National workshop "Indo-US Knowledge Initiative on Agriculture- Whither India Farmers?" Centre for Sustainable Agriculture \& Centre for World Solidarity, 8-9 Hyderabad December 2006.

[16] Ghosh, P.K. IPR issues in biotechnology in India and other developed countries. Invention Intelligence ISSN: 0970-0056 December 2001, 36(6), pp. 246-261

[17] The Geographical Indication of Goods (Registration \& Protection) Act 1999. No 48 of 1999. 30 December 2001 (www.patentoffice.nic.in/ipr/gi/geo_ind.htm)

[18] Group of Latin American Countries in the Caribbean (GRULAC) "Traditional knowledge and the need to give it adequate IP protection" WO/GA/26/9 14 September 2000.

[19] WTO committee on Trade and Environment Council for TRIPS (2000). Protection of Biodiversity and Traditional Knowledge: The Indian Experience Submission by India 2000.

[20] Gopalakrishnan, N.S. How to operationalize the disclosure requirement at the national level in a manner supportive to the TRIPS Agreement and the CBD? Centre for IPR Studies, School of Legal Studies, Cochin University of Science \& Technology, Cochin, Kerala, India

[21] Protection of Biodiversity and Traditional Knowledge. The Indian Experiences. WT/CTE/W/156, IP/C/W/198 14 July 2000.

[22] Www.nbaindia.org/docs/simplified_methodology_onphr.pdf

(C) Dipak B. Shukla; Licensee Bentham Open.

This is an open access article licensed under the terms of the Creative Commons Attribution Non-Commercial License (http://creativecommons.org/licenses/by-nc/3.0/) which permits unrestricted, non-commercial use, distribution and reproduction in any medium, provided the work is properly cited. 